\title{
Más allá de las políticas sociales ¿Hacia sistemas públicos de cuidados en el MERCOSUR?
}

\author{
Beyond social policies Towards public care systems in MERCOSUR?
}

DOI: https://doi.org/10.22456/2178-8839. 113760

Silvia Lilian Ferro

Universidade Federal de Integração Latino-americana (UNILA), Foz do Iguaçu, Brasil

lilian.ferro@umila.edu.br

\section{Resumen}

Esta comunicación se propone dimensionar consecuencias demográficas, económicas y sociales de la confluencia de la crisis sanitaria global provocada por la pandemia de COVID-19 desde marzo del 2020, con la preexistente y estructural crisis global del cuidado; recortando como espacio de análisis a países del MERCOSUR: Argentina, Brasil, Paraguay y Uruguay. Seguidamente, se hace un diagnóstico en perspectiva de género sob re políticas públicas de cuidados existentes en la región, en vista de las mudanzas laborales y familiares percibidas desde las últimas décadas en Occidente. Por último, se dimensionan insuficiencias de origen e inadecuaciones históricas de políticas sociales vigentes y amplificadas en el escenario pandémico. La metodología utilizada es mixta, con uso de técnicas cuantitativas y cualitativas en el procesamiento de información e interpretación de datos estadísticos, colocándolos en diálogo con la literatura de referencia en políticas y sistemas públicos del cuidado. Los resultados evidencian la necesidad de pensar en la generalización de sistemas públicos de cuidados como instancias superadoras de políticas sociales decimonónicas y originarias del Norte Global que dan señales de agotamiento de ciclo en sociedades del Sur Global y en especial en la región analizada.

Palabras clave: Políticas públicas; Cuidados; Políticas sociales; MERCOSUR;

\section{Abstract}

This communication aims to dimension demographic, economic and social consequences of the confluence of the global health crisis caused by the COVID-19 pandemic since March 2020 with the pre-existing and structural global care crisis; cutting as space for analysis MERCOSUR countries: Argentina, Brazil, Paraguay and Uruguay. Next, a diagnosis is made from a gender perspective on existing public care policies in the region, in view of the work and family changes perceived in the last decades in the West. Finally, inadequacies of origin and historical inadequacies of social policies in force and amplified in the pandemic scenario are measured. The methodology used is mixed, with the use of quantitative and qualitative techniques in the processing of information and interpretation of statistical data, placing them in dialogue with the reference literature on policies and public systems of care. The results show the need to think about the generalization of public care systems as overcoming instances of nineteenth-century social policies originating in the Global North that show signs of cycle exhaustion in societies of the Global South and especially in the analyzed region.

Keywords: Public policies; Care; Social policies; MERCOSUR; 


\section{Introducción}

A inicios del año 2020 irrumpe la crisis sanitaria causada por la pandemia del COVID-19, obligando a la Organización Mundial de la Salud (OMS) a declarar la emergencia sanitaria global. Esta situación coyuntural se encuentra con la preexistentey estructural crisis global de cuidados (PÉREZ OROZCO, 2006) que remite en sus inicios a un proceso histórico evidenciado en Occidente desde el último tercio del SigloXX y actualmente en difusión en gran parte del mundo. Se conjugaron factores significativos que tensionan arreglos consuetudinarios de organización social para el cuidado de las personas. Entre otros: a) incremento significativo y sostenido de la participación laboral y educativa femenina, b) transiciones demográficas, es decir caída progresiva de las tasas de fecundidad y simultánea expansión de la expectativa de vida, c) urbanización acelerada, crecimiento espacial de ciudades, sistemas de transporte públicos deficientes e insuficientes, d) nuclearización y verticalización de las familias y crecimiento de familias uniparentales jefaturadas por mujeres, asícomo homoparentales, e) dinámicas y condiciones laborales androcéntricas con cambios pocos significativos desde su modelización "industrial” a partir del Siglo XIX, f) insuficiente infraestructura pública de apoyo al cuidado y g) inexistencia de comprometimiento del sector empresarial (FERRO, 2020a).

Los cuidados se definen como la atención de las necesidades cotidianas de las personas en situación de dependencia, pudiendo ser realizadas por una persona remunerada o no remunerada, familiar o no familiar, en una institución o en el ámbito del hogar. El cuidado contiene un componente material y otro aspecto afectivo o vincular (AGUIRRE, 2008,p. 9).

Desde la deflagración pandémica, numerosos gobiernos adoptan medidas de enfrentamiento a la pandemia utilizando cuarentenas como herramienta generalizada para contener contagios, en tanto se mejoran a contra reloj infraestructuras sanitarias para responder a la emergencia. Confinan, en forma continua o alternada, a la mayor parte de la población en sus hogares restringiendo su circulación en el espacio público. Esta coyuntura intensifica las demandas de cuidados familiares, por ende, las asimetrías históricas y estructurales en la provisión de estos, que son considerados funciones y actividades propias de las mujeres de los núcleos de convivencia.

El cuidado es la garantía de supervivencia de la especie y a escala individual es una necesidad extendida temporalmente en el ciclo de vida de cada individuo. Tiene que ver con la vulnerabilidad paradojalmente mayor que tenemos en cuanto Homo Sapiens respecto de otros mamíferos, aun de aquellos menos “evolucionados” neurológicamente. Como primates superiores con diferenciales adaptativos claves, nos tornamos especie dominante en el planeta y al mismo tiempola especie más vulnerable corporal y emocionalmente (HARARI, 2018).

Desde el nacimiento hasta la mayoría de edad, la dependencia de cuidados para la sobrevivencia es total; por ejemplo, en la primera infancia, disminuyendo gradual y lentamente conforme el individuo va obteniendo autonomía en autocuidados, considerando aquí apenas aspectos psicomotrices y no autonomía económica para obtención de recursos que permitan proveerse de insumos, bienes y servicios necesarios. Aquellos individuos que superan con éxito la dependencia total de cuidados van obteniendo a la par que la autonomía personal, derechos jurídicamente esta blecidos formando parte de una ciudadanía. Pero que aún adultos y saludables, no pueden suprimir la posibilidad eventual de dependencia, forzosa o electiva, parcial o absoluta de cuidados a ser provistos por otra persona.

Por tanto, es el acceso al cuidado lo que garantiza la vida humana y no apenas el nacimiento. Los hogares son escenarios de la mayor prestación de servicios de cuidados no remunerados y la distribución de responsabilidades de su provisión continúa basándose en asimetrías emergentes de una organización social con impronta patriarcal, moldeada por expectativas sociales de comportamientos diferentes para hombres y mujeres, justificadas en la diferencia sexual y que legitima ámbitos de actuación públicos y privados, así como reconocimientos no simétricos y jerárquicos (SCOTT,1986).

Tales rasgos arcaicos todavía evidentes en nuestras sociedades contemporáneas tendrían origen según Saffioti (2004), en una rígida división sexual del trabajo característica de sociedades que habrían surgido hace por lo menos entre 
6.000 o 7.000 años. Este orden de cosas fue persistentemente contestado por algunas mujeres principalmente ${ }^{1}$ que se expresaron tanto en forma individual como integrando movimientos sociales y otras formas de acción colectiva. El impacto producido por movimientos feministas surgidos con la propia Modernidad, en todos los espacios de debate: política, ciencia, religión, derechoy cultura fue significativo (AMORÓS, 2005) y continúa desplegándose, intentando desmontar en forma definitiva tales resabios patriarcales. Esta tarea es dificultosa debido a que ideas y fundamentalmente emociones patriarcales, conservan todavía una porción significativa de consenso social.

En el plano académico, al estudio de relaciones asimétricas y jerárquicas entre hombresy mujeres, históricamente constituidas, con influencia directa en el acceso a oportunidades de desarrollo personaly al reconocimiento diferencial de lo contribuido por unas y por otros, en todas y cada una de las actividades humanas, se conoce com o enfoque de género. Según Scott (1986) el género es una categoría epistemológica relacional, dinámica e histórica, así como también una metodología de análisis de información.

La crisis sanitaria en curso pone en evidencia la injusta organización social de los cuidados ya que en el mundoy en especial en América Latina y el Caribe, hombres de diferentes estratos sociales y etnias, no asumen paritariamentelos trabajos y responsabilidades de cuidados (CEPAL, 2020). Las cuarentenas, extendidas temporal y espacialmente, exacerban conflictos y tensiones domésticas debido a la asimétrica distribución de los trabajos de cuidados y responsabilidades familiares en general entre hombres y mujeres, expresándose en muchos casos como violencia doméstica y de género. Esto hace inevitable su consideración pública en el actual contexto (HIRATA; GUIMARÃES, 2020).

Lo que están señalando estudios realizados por organizaciones internacionales multilaterales, gobiernos y organizaciones de la sociedad civil de los países seleccionados para este estudio (OMS, 2020; UN WOMEN, 2020; UNFPA,2020; REPUBLICA ARGENTINA, 2020; ARTICULACION FEMINISTA MARCOSUR, 2020; REPUBLICA ORIENTAL DEL URUGUAY, 2020) es que tensiones domésticas y en relaciones interpersonales están desembocando más frecuentemente en feminicidios, así como otras formas de violencia doméstica y de género; infelizmente ya en crescendo antes de la pandemia, aunque agravadas por ella.

Aquí se considera que las consecuencias más profundas de la crisis global de cuidados son m enos evidentes que el problema histórico de la violencia de género. Esta comunicación se propone visibilizarlas, aprovechandola centralidad que adquiere la cuestión del cuidado en el marco de la pandemia, apuntando a la confluencia de situaciones críticas de diferentes escalas, una estructural: crisis global de cuidados que se manifiesta con fuerza desde finales del sigloXX yllega hasta el presentey otra coyuntural: crisis sanitaria global deflagrada en 2020.

El actual escenario permite realizar observaciones en diferentes niveles, de los cuales se seleccionaron los siguientes:1) ponderación de adecuación de respuestas desde el sector público, es decir de políticas públicas de cuidados existentes en la escala regional MERCOSUR, normalmente consideradas dentro del área de políticas sociales, 2) binarización jerárquica entre el mainstream de políticas públicas respecto del subgrupo denominado políticas sociales, satelitales respecto de las primeras y con señales de agotamiento de ciclo en este escenario pandémicoy 3 ) potencialidad de la generalización de sistemas nacionales de cuidados como respuesta permanente y axial para el sostenimiento de condiciones de vida de las poblaciones.

El objetivo es ofrecer herramientas teóricas con fundamentación estadística para estimular el debate colectivoy compromiso de decisores públicos y privados, en sociedades cada vez más complejas y menos amigables con la distribución universal y equitativa del bienestar.

Se realizó una sistematización de alcances y posibilidades de políticas de cuidados en escala regional dentro del ámbito MERCOSUR, colocando en diálogo información estadística de organismos internacionales con conceptos y análisis surgidos de literatura de referencia en sistemas y políticas de cuidados en América Latina y de la economía feminista. Se

\footnotetext{
${ }^{1} \mathrm{Y}$ también por algunos hombres con sentido de justicia, aunque menos visibles históricamente.
} 
crearon matrices de variables e indicadores genéricos para dimensionar el déficit de sistemas públicos de cuidados, que puedan ser aplicados en específico a cada caso nacional de interés, más allá de los aquí modelizados. Se priorizó una selección de indicadores para análisis comparativo, siendo esta comunicación parte de una investigación mayor y bajo dirección de la autora. Se proponen, además, prospectivas en base a proyecciones demográficas para posibilitar a col ectivos sociales y decisores públicos, pensary actuar en escenarios concretos del cuidado en el futuro próximo.

\section{Gestación de una crisis sistémica}

Desde la segunda mitad del siglo pasado, la incorporación maciza de mujeres de sectores medios a estudios superiores y a mercados laborales, el impacto en la reproducción humana del desarrollo de tecnología química y farmacéutica para contracepción, los cambios en la conformación de las familias e influencia en el debate público de movimientos sociales reivindicando autonomías individuales decisorias, asícomo reconocimiento de la diversidad sexual e igualdad de oportunidades entre hombresy mujeres en todos los planos de actuación, fueron vectores de modernización cultural modificando irreversiblemente expectativas y comportamientos de hombres y mujeres en relación a proyectos y objetivos de sus vidas, acelerando transformaciones en estructuras socioeconómicas.

Estas transformaciones presentan desafíos inéditos a estados nacionales. Las mudanzas en la gestión del tiempo, por la distribución diaria del mismo entre responsabilidades familiares, movilidad entre hogares y ámbitos de actuación extra domésticos - como trabajo remunerado y/o estudios- acarrearon una creciente tensión en los hogares: "La conciliación entre los tiempos de cuidados y las exigencias del trabajo mercantil implican cada vez más, mayor movilidad y disponibilidad horaria de trabajadoras y trabajadores" (CARRASCO, 2001, p.1).

La expansión continua de la asalarización promovida por la industrialización en sus diferentes etapas históricas, intensificó la separación entre el hogar y el lugar de producción de bienes y servicios mercantiles; propiciando la concentración de gran parte de la población en ciudades donde están localizados grandes centros productivos,educativos, religiosos, así como la mayor parte de instituciones estatales y del poder social en general. El tiempo diario de mujeres y hombres, es medido en días terrestres de 24 horas, es decir tiene igual extensión para ambos sexos. Sin embargo, la ejecución de diferentes tareas en simultáneo que muchas mujeres practican para dar cuenta de dobles o triples jornadas de trabajo, parece estirarlo en varias horas a más para ellas, comoinforman estadísticas de usos d el tiempoen gran parte del mundo occidental (DARCY, 2003). Esta situación desembocó en un conflicto generalizadoy estructural entre la esfera de producción mercantil, situada mayormente fuera de los hogares, ampliamente valorada y estimulada a alcanzar s u máximo posible y la esfera del cuidado de las personas situada mayormente en hogares y no reconocida como relevante.

La progresiva nuclearización de las familias (JELIN,2010) tanto por separación geográfica de sus integrantes debido a migraciones internas, internacionales y hasta intraurbanas, como por cambios en expectativas culturales de socialización familiar, entre otras causas; dificulta utilizar el significativo recurso de cuidados gratuitos provistos por otras mujeres del grupo familiar ampliado, como ocurrió por generaciones y todavía se hace en América Latina, aunque más limitado a ciudades pequeñas y zonas rurales.

En Brasil, en 2016, las mujeres dedicaron un $73 \%$ más de horas al cuidado de personas y/o tareas domésticas que los hombres (18,1 horas frente a 10,5 horas). [...]El corte por color o raza indica que las mujeres negras y morenas son las que más se dedican al cuidado de las personas y/o quehaceres del hogar, con un récord de 18,6 horas semanales en 2016 [...] el indicador varía poco para hombres al considerar el color, la raza o la región de residencia. (IBGE, 2018, p.3)

La justificación más extendida para el rechazo o resistencias de la mayoría de los hombres para dividir paritariamente con las mujeres trabajos de cuidados de dependientes familiares, además de exigir ser cuidados por ellas estando aptos para cuidarse a símismos (PICCHIO,2001), tiene que ver con consensos patriarcales, que colocan el cuidado 
de otros como parte de la "naturaleza” femenina. Desentenderse de los mismos, libera tiempos y energías para dedicarse a tiempo completo a tareas y funciones consideradas propias de la "naturaleza" masculina.

Cuadro 1- Tiempo medio (en horas semanales) destinado al trabajo remuneradoy no remunerado, población encima de 15 años por sexo y país. Último período disponible ${ }^{2}$

\begin{tabular}{|c|c|c|c|c|}
\hline \multirow{2}{*}{ País } & \multicolumn{2}{|c|}{ Mujeres } & \multicolumn{2}{c|}{ Hombres } \\
\cline { 2 - 5 } & T. R. & T.n.R & T. R. & T.n.R \\
\hline Argentina & 15,2 & 42,4 & 33,2 & 17,3 \\
\hline Brasil & 16,8 & 21,5 & 28,6 & 10,8 \\
\hline Paraguay & 18,1 & 28,5 & 35,4 & 11,8 \\
\hline Uruguay & 21,8 & 35,7 & 36,8 & 15,4 \\
\hline
\end{tabular}

Fuente: Autora en base a CEPAL (2019).

Por trabajo de cuidados se entiende aquí al conjunto de prestaciones, tanto individuales, como grupales y colectivas que generan servicios altamente personalizados y que demandan inmensas inversiones de tiempos, energías físicas y especialización ${ }^{3}$. Estos servicios garantizan el bienestar biológico, psicológico, social y espiritual de las personas que se benefician de ellos; sean prestados en forma remunerada como gratuita, sean prestados en hogares como en espacios públicos (FERRO, 2019b). Su dimensión económica, más allá de la social, política y cultural es tan evidente como poco abordada: “desde una perspectiva socioeconómica, al menos para la economía oficial el sostenimiento de la vida no ha sido nunca una preocupación analítica central” (CARRASCO, 2001, p.5).

Habitualmente, conceptos como "trabajo de cuidados" y "trabajo doméstico" aparecen como dimensiones diferentes del trabajo no remunerado y remunerado que se realiza domiciliariamente. En la línea teórica de esta investigación se consideran ambas dimensiones incluidas enla primera porque, por ejemplo, la limpieza del hábitates una necesidad de higiene y ella es un elemento fundamental de preservación de la salud, esencial además para la calidad de vida. Además, la separación binaria, e innecesaria, entre trabajo doméstico y de cuidados causa no pocos problemas de registroy cuantificación:

Especialmente en países como Brasil, Colombia, Argentina y Uruguay, son las trabaja doras domésticas (trabajadoras mensuales o diaristas), antes que las profesionales del cuidado, las que se encargan, en gran medida, del cuidado infantil y de los mayores en el seno familiar. De modo que sería imposible comprender el crecimiento de las ocupaciones de cuidado en nuestros países si lo disociamos del papel desempeñado por el reclutamiento, para tales actividades, de trabajadoras domésticas, por regla general no clasificadas como trabajadoras de cuidado en las estadísticas oficiales (GUIMARÃES; HIRATA 2020, p.15).

En el debate público, la centralidad del cuidado en la organización social y su contribución a la sostenibilidad de todas las actividades que proveen bienes y servicios necesarios para la vida humana (PICCHIO, 2001) también fue persistentemente invisibilizada. Una de las consecuencias de la contradicción entre modernización de las sociedades impulsada por la tecnologización de la vida cotidiana y a su vez supervivencia de patrones patriarcales arcaicos en la

\footnotetext{
2 Por "último periodo disponible" se entiende que las mediciones pueden tener distintos años para cada país considerado.

3 La especialización del trabajo de cuidados es una cuestión debatida, porque tal palabra connota saberes técnicos o científicos acreditados. Aquí se refiere a la personalización basada en el profundo conocimiento deldestinatario de tales servicios. Conocimiento frecuentemente propiciado por lazo afectivos.
} 
división de responsabilidades y trabajos de cuidados familiares: "trabajo necesario para la reproducción del bienestar cotidiano y al cuidado de dependientes y de los propios trabajadores" (RODRÍGUEZ ENRIQUEZ, 2011, p.7), fueron las fuertes caídas de tasas de fecundidad en países de sarrollados y también en aquellos en vías de desarrollo. Argentina y Uruguay evidencian una caída más antigua y paulatina, encontrándose cerca del promedio regional latinoamericano, en tanto que Brasil y Paraguay manifiestan una caída más reciente y abrupta, siendo Brasil quien tendrá la menor tasa de natalidad hacia el quinquenio 2050 -55 según proyecciones y comparando con los demás países analizados (UN DESA, 2020).

Las transiciones demográficas ${ }^{4}$ son la respuesta silenciosa a la ausencia de debate colectivo sobre una nueva forma de organizar y distribuir responsabilidades sistémicas e interpersonales del cuidado de personas dependientes en diferentes estadios del ciclo de la vida, sumándose al problema la limitada e insuficiente respuesta desde las polít icas públicas y del sector privado. Las familias de sectores medios, mayoría entre familias demandantes de estos servicios de apoyo a los cuidados- por ser paradigmáticas del acceso femenino a mercados laborales y a la educación superior contornean este problema comprando esos servicios a mujeres más pobres. Aun cuando el trabajo de cuidados remunerado es el sector laboral más precario, de peores salarios y condiciones de trabajo, continúa siendo oneroso en el presupuesto familiar, especialmente en sociedades del Norte global donde derechos laborales de trabajadoras domésticas están más consolidados que en las del Sur Global (DURÁN, 2011). La solución fue promover la entrada de inmigrantes oriundas del Sur global para realizar esas tareas con salarios más bajos y sin la formalización laboral esperable en los casos de mujeres de la misma nacionalidad. (PARREÑAS, 2015).

Esas transformaciones están presentes también en países latinoamericanos, aunque con algunas características diferentes de las que experimentaron sociedades del Norte Global. Sin embargo, estas cuestiones fueron confinadas a espacios familiares y en el plano de relaciones interpersonales, como si fuesen apenas cuestiones éticas, afectivas o individuales, cuando en realidad: "Tales tareas constituyen la base de la sociedad puesto que generan trabajadores sanos y, al no ser remuneradas, constituyen ese motor invisible y barato que permite el rodaje económico del país" (ESPINO, 2001, p.15).

\section{Respuestas desde las políticas}

Es el cuidado- primariamente y en confluencia con otros recursos - lo que posibilita la existencia y reproducción de la población en cualquier país y es precisamente la existencia de población, primera condición material junto con un territorio para la existencia de estados nacionales. Por tanto, es una cuestión de Estado regular la provisión de cuidados con calidad, distribuirlo sistémicamente con atención a la equidad entre prestadores institucionales e individuales y garantizar una apropiada infraestructura pública de acceso universal.

La "prehistoria" de las actuales políticas de cuidados fueron las políticas maternalistas que se expresaron en el mundo laboral asalariado como protección a la maternidad de trabajadoras desde finales del siglo XIX en el marco del ideario político natalista, es decir estímulos a la procreación implementadas en la mayor parte de los estados occidentales (FERRO, 2019a) y que llegan hasta nuestros días resignificadas en objetivos y alcances, aunqueigualmenteinsuficientes y desarticuladas. En ellasla función a proteger era de procreación strictu sensu y no la calidad del cuidado brindado.

En la actualidad, con el crecimiento poblacional sostenido por décadas, la preocupación pública y política comienza a enfocarse en la incidencia del cuidado para el sostenimiento de la calidad de los procesos vitales individuales. En ese sentido por políticas de cuidados se entiende también la función de articulación en la provisión de estos por parte del poder público:

\footnotetext{
${ }^{4}$ La transición demográfica es la transformación de la estructura poblacional, ocurrida por caída persistente de nacimientos y progresiva expansión de la expectativa de vida, aumentando la población anciana en forma expresiva.
} 
La forma en la que una sociedad resuelve la demanda de cuidados resulta en una configuración denominada organización social del cuidado en la que el Estado, el mercado, las familias, la comunidad, los varones y las mujeres tienen un rol como proveedores. El Estado tiene un doble rol, al ser proveedor, pero al mismo tiempo ser quien asigna y regula la responsabilidad y la intervención de cada uno de los demás agentes (BATTHYÁNY et.al, 2017, p.297).

Siguiendo a Batthyány et.al. (2017) las políticas de cuidados existentes, aun cuando no utilicen ese nombre, pueden clasificarse según el criterio de locación de recursos pudiendo ser estos de tiempo, ingresos y servicios. Por políticas de asignación de tiempo se entienden las políticas públicas que reconocen o amplían derechos de tiempos remunerados fuera de espacios laborales para ser destinados a cuidados de dependientes familiares, de aplicación en el empleo público y privado.

En relación con las políticas de tiempo para cuidar, se trata de prestaciones que liberan tiempo del empleo para dedicarlo a los cuidados no remunerados (permisos de maternidad y paternidad, permisos de lactancia, excedencias por cuidados de familiares, reducciones de jornada, etc.). Pueden ser o no remuneradas, al igual que el tiempo liberado del empleo puede o no seguir contabilizándose como tiempo aportado a los seguros sociales\{...\}La mayoría de estas medidas están reconocidas por igual para mujeres y hombres, pero son derechos ejercidos casi en su totalidad por mujeres. La excepción es el permiso de paternidad que en muchos países no está reconocido y que, en caso de estarlo, es de una duración totalmente desproporcionada al de maternidad (REPÚBLICA DEL PARAGUAY,2019,p.10).

Aplicando esas definiciones a los casos nacionales seleccionados, podemos notar asimetrías de licencias maternales y paternales en el momento del nacimiento, más visibles en el debate colectivo porque son un obstáculo evidente a la mayor asunción paterna de responsabilidades de cuid ados. En Argentina estas licencias son establecidas a partir de la Ley de Contrato de Trabajo (LCT) de 1974 donde se establece; "la licencia por maternidad 90 días (menos de lo establecido por la OIT), por paternidad (2 días), el período de excedencia y de lactancia, la protección de la trabajadora embarazada, las licencias maternas por hijos con síndrome de Down" (BORGEAUD-GARCIANDÍA, 2020, p.38).

En Brasil la situación es más variada, si bien el permiso maternal por nacimiento es de 120 días, superandoincluso la recomendación de OIT de un mínimo de 98 días, establece en cambio un permiso paternal de 5 días, por debajo de países como Paraguay que otorga actualmente dos semanas posteriores al parto, con goce de sal ario a cargo del empleador, siendo dicho permiso de carácter irrenunciable (Art.13 Ley N ${ }^{\circ}$ 5.508/2015, Decreto Reglamentario N. ${ }^{\circ} 7550 / 2017$ ). En esta misma "Ley de promoción, protección de la maternidad y apoyo a la lactancia materna" paraguaya, la licencia materna es de 18 semanas, lo cual supera también al mínimo recomendado por OIT, concluyendo que este país es el mejor posicionado en el MERCOSUR al respecto.

En Uruguay se da el caso particular que en el ámbito privado las licencias maternales y paternales son más extensas respecto del empleo público. En el caso de trabajadoras madres del ámbito privado se reconocen con goce de haberes 14 semanas para el cuidado del recién nacido (Ley 19.161) y en el ámbito público son 13 semanas (Ley 19.121) $)^{5}$. Los trabajadores padres del ámbito privado, amparados por el Banco de Previsión Social (BPS), cuentan con licencia de 13 días y en el caso de la administración central son 10 días continuos, ambos casos con goce de haberes.

Por políticas de locación de ingresos se entienden todas las que estimulan, por vía salarial o de deducciones impositivas, la compra de bienes y servicios destinados a cuidados de dependientes familiares, por ejemplo, los ya mencionados salarios familiares, los pagos especiales por nacimiento, adopción, atención a la discapacidad de dependientes, etc. El salario familiar tiene una más larga tradición en todo Occidente que las deducciones impositivas. Impulsado por la Iglesia Católica a partir de su Encíclica Rerum Novarum de 1891, fue asumido debido a su influencia entre

\footnotetext{
${ }^{5}$ Una vez finalizada la licencia por maternidad, las trabajadoras del sector público y privado tienen derecho a reducir por lactancia a la mitad la jornada laboral hasta un máximo de 9 meses. El BPS cubre el 100\% del pago de las horas reducidas en el segundo caso. En caso de nacimientos múltiples, pretérminos o en caso de que el/la bebé tenga alguna discapacidad, la licencia por maternidad será de 18 semanas. En la adopción (Leyes 17.292 y 18.436) las prestaciones de licencias maternales y paternales son diferenciales respecto de las anteriores, pero indistintas según sexo del progenitor(REPÚBLICA ORIENTAL DEL URUGUAY, 2020a).
} 
las reivindicaciones de organizaciones sindicales promediando el siglo XIX e implantado por los estados por las mismas razones y según la lógica androcéntrica de su concepción: pagados al obrero/trabajador asalariado, a la vez considerado unipersonalmente jefe de familia. Llegó con ese formato a la regulación estatal de las relaciones laborales y a los mecanismos de protección social para el trabajo formalizado en países latinoamericanos, primero en los "vanguardistas" como Argentina y Uruguay (FERRO, 2019a) y después generalizados en diferentes grados de capilaridad al conjunto de países.

Por políticas de locación de servicios, también conocidas como "de respiro familiar", se entiende a la infraestructura pública que ofrece servicios de cuidados permitiendo delegar parte de ellos desde familias hacia instituciones, por ejemplo: jardines de infancia, hogar de ancianos, centros de día, instituciones para la discapacidad, cuidados especiales en ámbitos educativos, hogares de huérfanos, casas-cuna y espacios de inclusión en general. Este tipo de políticas están focalizadas en la primera infancia y aun asíson insuficientes en cuanto a su demanda en los cuatro países analizados aquí.

El caso brasilero es destacable porque la guardería es un derecho constitucional desde 1988 "de los y las trabajadoras con hijos de 0 a 5 años como de los niños; es decir, simultáneamente, un derecho al trabajoy a la educación” (GUIMARÃES et.al;2020, p.102). Tal estímulo influenció en la expansión de infraestructura, aunque las autoras evidencian que no alcanza a cubrir la demanda especialmente por parte de sectores populares. Esta insuficiencia en la provisión de servicios públicos suficientes para atender necesidades de cuidados en la primera infancia, en discapacidad de cualquier edad y especialmente en la vejez está sostenida por consensos sociales comunes en los cuatro países según informan estudios citados aquí, estableciendo que el cuidado extra doméstico debería ser última opción y después de agotarse instancias maternas o de otras mujeres de la propia familia o remuneradas por ella, especialmente en el caso de adultos mayores. Todo esto resulta en un sesgo fuertemente mercantilizado de este tipo de servicios, mostrando por el lado de la oferta un perfil de prestadoras pobres, afrodescendientes en su mayoría y con altos niveles de precarización, informalidad y subregistro ${ }^{6}$; y por el lado de la demanda fuerte concentración de los sectores medios y altos. Para las familias pobres los servicios de cuidados accesibles son los ofrecidos por el sector público que tiene en su haber políticas de cuidados, aun cuando no asuman esa denominación, iniciadas promediando el siglo anterior y más intensamente desde los inicios del actual, aunque no integradas sistémicamente:

En condiciones demarcadas por la ausencia (o fragilidad) de la asistencia social y la imposibilidad (o dificultad) de recurrir al mercado de servicios de cuidados, ni el Estado (mediado por las políticas de asistencia) ni el mercado (al cual se accede por medio del poder de compra) se constituyen en alternativas para proveer el cuidado de personas. Entre los más pobres, las redes de reciprocidad aseguran dicha provisión, sostenidas por el apoyo comunitario o la familia extendida (GUIMARÃES $e t$ al; 2020,p.98-99).

Desde los inicios del siglo en curso, países como Uruguay, Chile y Costa Rica comenzaron a incorporar el enfoque sistémico y transversalizado en sus políticas públicas para la provisión de servicios de cuidados, siendo Uruguay el país más avanzado en ese sentido- tanto en comparación con países del MERCOSUR, como latinoamericanos en generalcreando un Sistema Nacional Integral de Cuidados (SNIC) el cual articula políticas de cuidados ya existentes, ampliándose en alcance y capilaridad social. Argentina, país que se caracteriza por una gran participación de las organizaciones de la sociedad civil en la prestación e infraestructura pública de servicios de cuidados, está comenzando a articular estas políticas desde el recientemente creado Ministerio de las Mujeres, Géneros y Diversidad (diciembre de 2019).

\footnotetext{
${ }^{6}$ Rasgo común en toda la región a escala latinoamericana.
} 
Paraguay cuenta con un Documento Marco para la elaboración de la Política Nacional de Cuidados impulsado por el Poder Ejecutivo. Esta política se encuentra en fase inicial y allíse diagnostica que:

\begin{abstract}
La normativa vigente presenta limitaciones para garantizar plenamente el derecho al cuidado. En primer lugar, al estar centrados en los aspectos biológicos de la maternidad, el alcance de estas regulaciones se circunscribe solo al periodo de tiempo posterior al nacimiento. En segundo lugar, protege fundamentalmente a la madre asignando casi exclusivamente el rol del cuidado a la mujer, lo cual a su vez limita su potencial como mecanismo para promover una mayor corresponsabilidad masculina. En tercer lugar, el Código laboral y la Ley Nro. 5.508 regulan fundamentalmente el trabajo formal, por lo tanto, su cobertura es limitada no solo porque beneficia a la población económicamente activa, sino que además a las que trabajan en relación de dependencia. En el caso de las mujeres, principales titulares de estos derechos, esto significa una cobertura menor al $20 \%$ del total en edad de trabajar. Finalmente, las deficiencias en la fiscalización tienen como resultado la baja cobertura de algunos de los derechos establecidos, como el del establecimiento de espacios para el cuidado infantil en las empresas de 50 o más trabajadores/as (REPÚBLICA DEL PARAGUAY, 2019,p.14 -15).
\end{abstract}

La limitación más importante de las políticas de cuidados existentes en los países analizados es la orientación hacia potenciales beneficiarios del cuidado, excluyendo regularmente de su consideración normativa y programática derechos y reivindicaciones de quienes cuidan, a excepción de Uruguay quien avanza también en ese sentido.

\title{
De políticas sociales hacia políticas de cuidados
}

Las políticas de cuidados existentes suelen considerarse como parte de las llamadas políticas sociales. Tácitamente, se entiende con esta forma de adjetivar políticas públicas que apenas una parte de estas: las "sociales", estarían enfocadas en el bienestar de los sectores más vulnerables de las sociedades; es decir "políticas para pobres” frecuentemente con pobres presupuestos también- esto tácitamente implica que las demás políticas públicas, sin el adjetivo de "sociales" se asumen orientadas a grupos más favorecidos. En la perspectiva teórica sustentada por este trabajo, las políticas sociales poseen restriccionesy limitaciones propias del paradigma social y político que las originóy la pandemia actual contribuye a profundizary visibilizar.

A lo largo del sigloXIX, maduróla Revolución Industrial iniciada a finales del sigloXVIIIy fue ya evidente que, sin protección estatal sobre las condiciones de trabajo gran parte de la población asalariada y de aquellos considerados sus dependientes familiares, quedaban desprotegidos ante eventual pérdida forzosa de ingresos del asalariado. Las revoluciones liberales en Europa, a partir de 1848 (HOBSBAWM, 2010) dieron origen a sindicatos y diversas formas de representación de intereses de obreros y trabajadores asalariados en general, obteniendo conquistas significativas con mucha movilización política.

A consecuencia de ello, a finales del siglo XIX, en algunos países europeos se crean sistemas de protección social de la población trabajadora que se materializan en la forma de legislación laboral y previdenciaria, extendiéndose a gran parte de Occidente.

Esta nueva forma de entender las relaciones políticas y económicas entre Estados, asalariados/as y empresas se tornó más tarde, paradigma de acción pública en el escenario de posguerra desde la mitad del siglo XX, surgiendo así Estados y Regímenes de Bienestar. Estas formas estatales elevaron al primer plano político e institucional, las llamadas políticas sociales que intentan proteger aquella parte de población que tiene una relación desigual de poder económico y político con sus empleadores, no contando por parte de estos con adecuadas protecciones ante las contingencias vitales que impiden la consecución regular de la prestación remunerada de sus servicios como enfermedades, accidentes incapacitantes, vejezy desempleo ocasional, entre otras.

En el escenario latinoamericano, la implantación de políticas protectoras de poblaciones vulnerables tuvo un curso más limitado y tardío que en Europa, a excepción de algunos países como Argentina y Uruguay que las inician también a finales del siglo XIX. En gran parte de América Latina, las llamadas políticas sociales se superponen sobre 
desigualdades estructurales configuradas históricamente, las que consolidaron exclusiones típicas de sociedades coloniales: "Detrás de la exclusión de derechos sociales se pueden encontrar los atributos de raza, color y lengua en todas las sociedades latinoamericanas." (RAMA, 2001, p.5). Otra razón que explic aría la baja adhesión de algunos países de la región a modelos de protección social típicos de Europa central, según este autor tendría que ver con la abundancia preexistente de mano de obra a diferencia del contexto europeo más industrializado.

En la década del ' 30 del siglo XX gobiernos de México, Brasil e Argentina iniciaron en forma pionera el camino de Industrialización por Sustitución de Importaciones (HALPERIN DONGHI, 2010) posibilitandola implantación posterior de Regímenes de Bienestar caracterizados por la creación, expansión y ampliación de políticas sociales.

Más adelante, políticas sociales surgidas desde el paradigma de la protección social de sectores asalariados, son legitimadas globalmente a partir del Pacto Internacional de Derechos Económicos, Sociales y Culturales sancionado en 1966-y en ejecución desde 1976- comprometiéndose los países signatarios a trabajar para la concesión de tales derechos a cada persona, incluidos derechos laborales, a la salud, a la educación y a un nivel de vida adecuado.

En los 70 estaría situado el final de las décadas "doradas" de los Estados de Bienestar en todo Occidente (HOBSBAWM, 2010). Cobran notoriedad teorías económicas neoliberales que fueron difundidas a través de medios de comunicación y de propaganda política, por tanto, escuchadas por las sociedades. Surgidas del núcleo de economistas conocidos como "Escuela de Chicago", consiguen instalar en la era de gobiernos militares en América Latina vía golpes de estado, la idea de que el Estado de Bienestar habría fracasado; sería una obsolescencia, un obstáculo a superar para así facilitar las nuevas vías del crecimiento económico en todo el mundo, protagonizadas por el sector financiero.

En la década de los 80, la “década perdida”, políticas de liberaliza ción de economías nacionales junto con cesación de pagos de deudas soberanas contraídas en la década anterior, afectaron em mayor medida a países del Sur global. Desindustrialización, desempleo, escaladas inflacionarias, violencia política y retrocesos en la distribución funcional del ingreso fueron consecuencia de la aplicación "exitosa" del programa económico-político neoliberal. Esto incrementó fuertemente la demanda de cuidados gratuitos tanto en hogares, como en la comunidad, sustituyendo por el "hacer en casa" la compra habitual de estos servicios: "Además, se estámodificandola distribución de responsabilidades respecto al bienestar de la población entre Estado, empresas, familias, personas, con consecuencias como el incremento de la inseguridad y de las tensiones domésticas” (PICCHIO, 2009, p.28).

En los ' 90 , se constatan las insuficiencias de políticas sociales tradicionales para contener el crecimiento exponencial de la pobreza en América Latina, región que no es la más pobre del mundo pero sí la más desigual (ONU HABITAT, 2010), no solo por el retiro del Estado de sus anteriores funciones distribuidoras, propiciadoras de igualdad y movilidad social ascendente; sino también porque el destinatario seguía siendo el hombre asalariado, el Male Breadwinner (FERRO, 2019a), en cuanto crecían, sin solución de continuidad, hogares jefaturados por mujeres, principalmente madres.

Organismos internacionales como Banco Mundial y Naciones Unidas descubren entonces, que en América Latina la pobreza crece feminizada, especialmente en hogares con niños. También CEPAL en 2017 informó que por cada 100 hombres viviendo en la pobreza había 113 mujeres en esa situación. En este contexto, tales organismoslanzaron programas de "alivio a la pobreza" financiando políticas focalizadas para ser aplicadas en el Sur global, llamadas Programas de Transferencias Condicionadas (PTC): “Estos programas buscan transformar y detener la transmisión intergeneracional de la pobreza mediante el desarrollo de capacidades humanas en lasfamil ias más vulnerables.” (CECCHINI; MARTÍNEZ, 2011, p.5). Son "condicionadas" porque a cambio de las transferencias monetarias, las madres ${ }^{7}$ deben garantizar acceso escolar y a cuidados sanitarios para los niños.

\footnotetext{
${ }^{7}$ Inicialmente se transferían los recursos a los hombres siguiendo la lógica del "jefe de familia", pronto fue claro que las madres como destinatarias garantizan más su uso para el bienestar familiar y particularmente de infantes (BATTHYÁNY, 2015).
} 
Otra de las consecuencias del empobrecimiento generalizado en la región, fue el fuerte estímulo para la inclusión de trabajadoras latinoamericanas en flujos migratorios internacionales extracontinentales y transnacionales ${ }^{8}$. Las rentas obtenidas por prestación de servicios de cuidados remunerados en otros países son remitidas en gran parte a sus familias de origen. Estas remesas constituyeron, junto a las del resto de trabajadores migrantes, la principal forma de obtención de divisas para algunos países latinoamericanos (BANCO MUNDIAL, 2018). Dividendos generados con gran costo emocional de migrantes y sus familias, llegando incluso a desestructurar comunidades enteras. Costos soportados también por mujeres que permanecen en el grupo familiar desmembrado, responsabilizándose, en sustitución de las females bread winners, de cuidar dependientes y parejas desempleadas.

A inicios del siglo XXI, una ola de gobiernos progresistas, también caracterizados como "neodesarrollistas” (DA SILVA, 2019), en América Latina, especialmente en el Cono Sur americano, llegan al poder gubernamental durante ciclos tan intensos como intermitentes debido a los límites que reacciones conservadoras, liberales y neoliberales les imponen. Protagonizan a lolargo del subcontinentey especialmente en el Cono Sur americano, las llamadas “ondas rosas” (PANIZZA, 2006) de las izquierdas latinoamericanas, que intentan reiniciar etapas reparadoras de desequilibrios y desigualdades sociales, mediante transferencias de ingresos y oportunidades para los quintiles más desfavorecidos. Esto significó en la práctica un nuevo auge de políticas sociales “clásicas”, aunque ahora combinadas con la nueva generación de PTC, posteriormente asumidas algunas en su financiamiento- parcial o integralmente- por los estados: por ejemplo, Bolsa Família en Brasil, Asignación Universal por Hijo/a en Argentina o Programa Oportunidades en México entre los más conocidos.

En escenarios de persistente informalización laboral, con gran parte de hombres y mujeres insertos en actividades productivas de economías sumergidas y de baja productividad, las PTC consiguen llegar a los "núcleos duros" de reproducción de pobreza: madres solas con muchos dependientes familiares.

Estas asimetrías en la distribución de responsabilidades familiares originadas en los hogares se proyectan a las organizaciones en la forma de segregaciones debido a género, verticales ${ }^{9}$ y horizontales, condicionando diferencialmente posibilidades de desarrollo profesionaly laboral, considerando tiempos, horarios, desplazamientosy viajes. Muchas veces esto resulta en autoexclusión de asalariadas y empresarias al acceso a cargos y funciones que podrían dificultar todavía más, su ya complicada "ingeniería del tiempo" (DARCY, 2003) y la pax doméstica. Esto también en conductas discriminatorias de contratación por parte de empleadores (GÁLVEZ,2006). Este nudo de desigualdad se origina en los hogares amplificándose en organizaciones laborales y en el espacio público en general y también se identifica en aquellos espacios de alta cualificación técnica e intelectual como organizaciones de representación política, sindical, religiosas, medios de comunicación, etc.

Cabe entonces preguntarse: ¿cómo está compuestala oferta desdela infraestructura pública de cuidados en cada sociedad?

\section{Aspectos estructurales para mensurar déficits nacionales del cuidado}

Por oferta de servicios de cuidado se entiende el conjunto de prestaciones de servicios de cuidados disponibilizados a la población, pudiendo ser provistos tanto en hogares como fuera de ellos, ser remunerados o no remunerados, tener carácter familiar, empresarial o institucional y comprenden la atención de necesidades vitales de cada individuo o grupo, para el cumplimiento de funciones biológicas y de adaptación social, a través del mantenimiento

\footnotetext{
${ }^{8}$ Paraguay tiene un claro perfil exportador de servicios de cuidados, hacia Argentina en primer lugar, secundariamente a destinos extracontinentales y en menor medida hacia Brasil y Uruguay (FERRO, 2020a).

${ }^{9}$ Esta situación es más marcada en el sector privado que en el público, aun cuando en ambos se observa, que las mujeres abundan en niveles basales, en tanto los hombres están sobrerrepresentados en niveles dirigenciales y de liderazgo, hasta en aquellas organizaciones que en términos absolutos son paritarias en composición por sexo y aun en las feminizadas.
} 
cotidiano del bienestar físico, intelectual, emocional y espiritual. También, en eventuales enfermedades, deficiencias, accidentes, vejez y cualquier otro impedimento del individuo para cuidar de sí mismo (FERRO, 2019a). La dimensión colectiva del trabajo de cuidados no remunerado se denomina voluntariado.

Para dimensionar el déficit de cuidados por el lado de la oferta, se brinda aquí esta herramienta de mapeo de la organización social de cuidados que puede aplicarse, por su diseñogenérico, a cualquier caso nacional.

Cuadro 2- Sistema de provisión de servicios de cuidados por tipo de prestadores

\begin{tabular}{|c|c|c|c|c|c|}
\hline \multirow{2}{*}{$\begin{array}{l}\text { Subsistemas / } \\
\text { tipologías }\end{array}$} & \multirow{2}{*}{$\begin{array}{l}\text { Cuidados } \\
\text { familiares }\end{array}$} & \multirow{2}{*}{$\begin{array}{c}\text { Cuidados } \\
\text { remunerados no } \\
\text { institucionalizados }\end{array}$} & \multicolumn{3}{|c|}{ Cuidados institucionalizados $^{10}$} \\
\hline & & & $\begin{array}{l}\text { Cuidados } \\
\text { estatales }\end{array}$ & $\begin{array}{l}\text { Cuidados } \\
\text { comunitarios }\end{array}$ & $\begin{array}{c}\text { Cuidados } \\
\text { empresariales }\end{array}$ \\
\hline $\begin{array}{c}\text { Local de } \\
\text { prestaciones }\end{array}$ & $\begin{array}{l}\text { Provistos enlos } \\
\text { hogares, } \\
\text { generalmente } \\
\text { entre personas } \\
\text { unidas por } \\
\text { vínculos de } \\
\text { parentesco. }\end{array}$ & \begin{tabular}{|c|} 
Provistos \\
generalmente en los \\
domicilios de los \\
demandantes.
\end{tabular} & \begin{tabular}{|c} 
Provistos en la \\
institucionalidad \\
pública, estatal, p. \\
e. sistema de \\
salud pública y \\
privada, en el \\
ámbito \\
educacional, en \\
casas de acogida y \\
de apoyos a \\
personas con \\
deficiencias, así \\
como en sistemas \\
prisionales, etc.
\end{tabular} & $\begin{array}{c}\text { Generalmente } \\
\text { provistos en } \\
\text { espacios extra } \\
\text { domésticos, en } \\
\text { locales de (o por } \\
\text { las) } \\
\text { Organizaciones } \\
\text { de la Sociedad } \\
\text { Civil: Iglesias, } \\
\text { Cooperativas, } \\
\text { Sindicatos etc. }\end{array}$ & $\begin{array}{l}\text { Generalmente } \\
\text { provistos en } \\
\text { espacios extra } \\
\text { domésticos, en } \\
\text { locales de (o por } \\
\text { las) empresas } \\
\text { especializadas en } \\
\text { servicios de } \\
\text { cuidados: p.e. } \\
\text { sistema de salud } \\
\text { privadoy } \\
\text { remuneradoy } \\
\text { casas de acogida } \\
\text { de un amplio } \\
\text { abanico de } \\
\text { demandas de } \\
\text { cuidados. }\end{array}$ \\
\hline
\end{tabular}

Fuente: basado en FERRO, $2020 b$

El cuidado de cada persona a lo largo de su vida es resultado de la combinación de cada subsistema, formal e/o informal mencionado aquí, "Particularmente, en nuestras sociedades occidentales industrializadas, la subsistencia y calidad de vida se nutre fundamentalmente de tres fuentes:las producciones y actividades de cuidados directos realizadas desde el hogar, el mercado y la oferta de servicios públicos” (CARRASCO, 2001, p.2). En términos cuantitativos y cualitativos, es mayor la participación de los hogares en la prestación de servicios de cuidados pro-bono. Sin embargo, según datos de CEPAL basados en la PNAD continua en el casobrasileiroy en Encuestas Permanentes de Hogares en los casos de Paraguay, Uruguay y Argentina; apenas el 13\% de las mujeres em media regional y actualmente en descenso expresivo, declararon actividad principal las “tareas domésticas”. El restante 87\% de ellas sostiene un difícil equilibrio entre provisión de cuidados familiares y empleoy/o educación formal.

Si el cuidado es una necesidad universal, su demanda también lo es. Para mensurarla prima facie se considera el total de la población. La temporalidad de los ciclos vitales puede contribuir a clasificar esta demanda como fija o eventual. Por demanda de servicios de cuidados, se entiende la sumatoria de necesidades de acceso a prestaciones de cuidados, alimentación, higiene corporal y del hogar- prestados dentro o fuera de los núcleos de convivencia, remunerados o no remunerados. Estas necesidades son decurrentes de vulnerabilidades intrínsecas a la dimensión animal humana, asícomo su dimensión cultural. Dentro de "necesidades" de cuidados sólo se consideran situaciones forzosas en las que un

\footnotetext{
${ }^{10}$ Aquí se concentran la mayor parte de las front lineoccupations, muy visibles actualmente en el sector sanitario, poco pagadas y desgastantes psíquica y emocionalmente por la cercanía de las interacciones entre prestadores y usuarios. No casualmente ampliamente feminizadas.
} 
individuo no pueda cuidar autónomamente de sí mismo, como edad avanzada, enfermedad, discapacidades físicas e intelectuales y accidentes, etc. y no a las exigencias de quienes eligen no hacerlo en atención a la cultura patriarcal predominante.

Un indicador útil para ponderar la gravitación de la demanda integral, efectiva y potencial, de cuidados, es la relación de dependencia demográfica: "una medida demográfica para expresar la relación de la población, en términos de edad, entre las personas potencialmente activas y las personas potencialmente dependientes (inactivas). Normalmente se utilizan tres medidas: relación de dependencia total, infantil y adultos mayores” (CEPALSTAT, 2019).

Cuadro No.3 Relación de dependencia demográfica por grupos dependientes. Comparación de proyecciones 2020-2050.

\begin{tabular}{|c|c|c|c|c|c|c|c|c|}
\hline \multirow{2}{*}{$\begin{array}{c}\text { Grupos } \\
\text { dependientes }\end{array}$} & \multicolumn{9}{|c|}{ País } \\
\cline { 2 - 9 } & \multicolumn{2}{|c|}{ Argentina } & \multicolumn{2}{|c|}{ Brasil } & \multicolumn{2}{|c|}{ Paraguay } & \multicolumn{2}{c|}{ Uruguay } \\
\cline { 2 - 9 } & 2020 & 2050 & 2020 & 2050 & 2020 & 2050 & 2020 & 2050 \\
\hline Total & 55,77 & 57,31 & 43,48 & 59,22 & 55,53 & 50,35 & 54,85 & 61,73 \\
\hline Infantil & 38,06 & 30,13 & 29,71 & 23,05 & 44,94 & 31,14 & 31,14 & 26,68 \\
\hline ancianos/as & 17,71 & 27,18 & 13,76 & 36,18 & 10,59 & 19,2 & 23,36 & 35,05 \\
\hline
\end{tabular}

Fuente: Autora en base a datos de CEPALSTAT

Una limitación importante de este indicador es que la relación de dependencia demográfica se distribuye simétricamente entre hombresy mujeres a partir de 15 años en cada país. Analizando otros indicadores, com o reparto del tiempo entre trabajo remunerado y no remunerado en la región aquí considerada (ver cuadro N.1) inferimos que los resultados de la tasa de dependencia por grupos poblacionales deberían distribuirse por sexo restando de los hombres su parte incumplida de cuidados de dependientes familiares, es decir que no dividen paritariamente con las mujeres. También habría que restar la parte de aquellos hombres que aun cuando son aptos física y psicológicamente para cuidar de sí mismos, exigen o esperan ser cuidados por las mujeres ${ }^{12}$ : "Un hombre adulto sano y trabajador insume tanto trabajo doméstico y de cuidado como un niño de 5 años. El problema no es sólo que no comparten paritariamente el trabajo domésticoy reproductivo, sino que no se cuidan a sí mismos” (PICCHIO, 2003, p.12).

Considerando porcentuales de trabajo no remunerado distribuidos por sexo en cada país, podemos inferir que la tasa de dependencia ajustada y en media regional, para mujeres y hombres podría estar cerca de la relación 70 -30 respectivamente. Estos ajustes en las mediciones correlacionando indicadores darían una aproximación más real de la "foto" de la asimetría.

Perspectivas demográficas en países del MERCOSUR destacan las disparidades, según datos de UN DESA y CELADE-CEPAL, en tanto países del área ya "gastaron” su bonus demográfico, otros pioneros en urbanización como Argentina y Uruguay, tienen su pico poblacional proyectado hacia 2080 e 2085 respectivamente, Brasil está en pleno uso de su bono demográfico y será el primero de los países considerados en alcanzar el pico poblacional hacia el 2045. A partir de allí experimentará una disminución menos brusca que la subida y Paraguay alcanzaría su pico poblacional hacia el año 2070.

\footnotetext{
${ }^{11}$ Dependencia total: cociente entre la suma de los grupos poblacionales (población 0-14 + población 65 y más), en el numerador, dividido por la población de 15-64 años, en el denominador, multiplicado por 100. Infantil: cociente entre la población de 0-14 años, en el numerador, dividido por la población de 15-64 años, en el denominador, multiplicado por 100. Ancianos/as: cociente entre la población con 65 años o más, en el numerador, dividido por la población de 15 a 64 años, en el denominador, multiplicado por 100.

${ }^{12}$ Algunas ancianas asumen el cuidado de sus parejas también ancianos y hasta de nietos u otros familiares dependientes, especialmente en sectores más pobres.
} 
Respecto de la caída de la tasa de fecundidad, fenómeno común en casi todo el mundo, países como Brasil y Paraguay muestran su caída en velocidad y profundidad significativas, a diferencia de Argentina y Uruguay donde el inicio de la caída es anterior y menos brusca. Lo que es regla, como a escala global, es el expresivo envejecimiento poblacional por ampliación de la esperanza de vida, el cual junto con la caída de tasas de fecundidad adquiere la forma gráfica de una “tijera”. Situación imperativa para realizar prospectivas que optimicen la organización de cada sociedad para atender el incremento exponencial de demanda de cuidados. "En Latinoamérica predomina la demanda de cuidados para la población infantil, pero el rápido envejecimiento de la población en varios países latinoamericanos muestra la necesidad de anticiparse...” (DURÁN, 2011, p.15).

Cuadro 4- Esperanza de vida al nacer, serie históricay proyecciones

\begin{tabular}{|c|c|c|c|c|c|c|}
\hline \multirow{2}{*}{ País/región } & \multicolumn{2}{|c|}{$1950-55$} & \multicolumn{2}{c|}{$2020-25$} & \multicolumn{2}{c|}{$2095-100$} \\
\cline { 2 - 7 } & $\mathrm{H}$ & $\mathrm{M}$ & $\mathrm{H}$ & $\mathrm{M}$ & $\mathrm{H}$ & $\mathrm{M}$ \\
\hline América Latina & 49,4 & 55,9 & 72,9 & 79,2 & 85,1 & 88,6 \\
\hline Argentina & 60,4 & 67,4 & 73,8 & 80,4 & 85,2 & 88,9 \\
\hline Brasil & 49,1 & 54,7 & 73 & 80,1 & 85,7 & 89,8 \\
\hline Paraguay & 60,7 & 65,2 & 72,6 & 76,8 & 82,6 & 85,2 \\
\hline Uruguay & 63,3 & 70,4 & 74,8 & 81,9 & 85,7 & 90,2 \\
\hline
\end{tabular}

Sin embargo, la urgencia en la creación de un mainstream público del cuidado no depende solo de la temporalidad de llegada del pico poblacional o la intensidad de su transición demográfica, sino también de la insuficiencia del punto de partida. Es el desafío de la hora para Paraguay, porque tendrá una transición demográfica más marcada con base en una población bastante pequeña, en comparación con los socios mayores del bloque.

\section{Conclusión}

La crisis sanitaria global pone en escena la importancia de los cuidados para nuestra supervivencia y la insuficiencia de infraestructura pública y privada para su provisión efectiva, aunque la discusión sobre este tema aparece reducida generalmente a aspectos sanitarios e institucionalizados como "acceso a la salud", apenas la punta del iceberg de cuidados implicados en el desarrollo de cada vida humana. La mayor parte de su provisión es gratuita y no reconocida en su importancia crucial en el plano político, económico, social y cultural ya que han sido reducidos como instintos de las mujeres.

La cuestión del cuidado está insertándose despacio, aunque inexorablemente, en las agendas regionales de políticas públicas porque las mudanzas estructurales están profundizándose y con ellas la necesidad de acompañar la reorganización de facto de las sociedades, con políticas públicas que lideren lo que ya está ocurriendo. Las políticas de cuidados integradas en sistemas nacionales transversales a todas las políticas públicas serían una instancia superadora de las políticas sociales, tanto de las tradicionales de alcance "universal”, como de aquellas focalizadasy complementarias de insuficiencias de las primeras, en vistas de conseguir una protección social integral y efectivamente universal.

La complejidad de formas de organización de la convivencia en nuestras sociedades actuales precisa colocar la corresponsabilidad de las prestaciones de cuidados en primer plano del interés colectivo, la cual excede a la justa demanda 
de división equitativa entre hombres y mujeres titulares de hogares, ligados o no por vínculos de parentesco. Incluye también al Estado, a las organizaciones de la sociedad civil y a las empresas. El sistema público debe profundizar, extender y sistematizar respuestas al déficit del cuidadoy el sector privado aparecer cuando menos en el debate público, incluyendo la cuestión del cuidado en su responsabilidad social, porque son usuarios directos de los resultados de cuidados familiares. Las capacidadesy potencialidades dela ciudadanía, que en gran parte también son trabajadores,empresarios y voluntarios sociales, fueron y son forjadas por trabajos de cuidados multidimensionales que hacen gratuitamente las mujeres en los hogares, con insuficiente participación de los hombres.

Independientemente de dinámicas poblacionales, aun cuando estas señalan prioridades, es urgente planear la respuesta a esta demanda porque comienzan a tomar fuerza en la región las reivindicaciones de equidad de género y sistémica desde gran parte de las cuidadoras de nuestras sociedades, especialmente en Argentina y Uruguay. Evidenciar resultados de mudanzas de paradigma en las políticas públicas por medio del mejoramiento de una infraestructura pública de servicios suficientes y de calidad para la población potencialmente demandante, lleva tiempoy tiene que considerar su utilización generacional futura por las siguientes generaciones.

La multidimensionalidad del concepto cuidado también permitiría integrar en los sistemas públicos de provisión, la dimensión no humana y ambiental, descentrando su connotación autorreferencialmente humana. El déficit del cuidado en cada país en sus diferentes escalas puedey debe ser cuantificado y calificado apropiadamente, desarrollando una matriz de variables e indicadores de componentes dela demanda y la oferta de servicios de cuidados públicos. Estova mucho más allá de cuidados sanitarios o institucionalizados que parecen ser los únicos visibles y reconocidos como tales.

La implementación de sistemas integrales de cuidados en cada país tiene directa relación con el consenso social que predomina en sus sociedades sobre el valor de la vida humana, independientemente de cualquier parámetro de merecimiento y condicionalidad. Si continúa predominando la idea que las personas tienen como destino primordial la producción de bienes y servicios, muchas veces tornándose sus propias vidas, apenas insumos de esa producción, cuidar de ellas tendría una importancia relativa. Merecerían ser cuidadas y consideradas en sus derechos como cuidadoras, así como beneficiarios de cuidados, en cuanto produzcan para el espacio mercantilizado. En cambio, si la calidad de los procesos de toda vida humana es un objetivo político y moral prioritario recostado en un consenso societal macizo, la producción de bienes y servicios será apenas un medio, más allá de su importancia, para contribuir a la felicidad humana. En un escenario así, cuidar en forma integral, justa, equitativa y democrática tiene todo sentido. Tal decisión es colectiva, política y moral.

\section{Referências}

AGUIRRE, R. El futuro del cuidado. In:ARRIAGADA, I. (Ed.) Futuro de las familias y desafíos de las políticas. Santiago de Chile: CEPAL, 2008 p.23-34

AMORÓS, C. Globalización y orden de género. In: AMORÓS, C; DE MIGUEL, A. (Ed.) Teoría Feminista: de la Ilustración a la Globalización. Minerva Ediciones, Madrid, 2005. p.301-332.

ARTICULACION FEMINISTA MARCOSUR. Tu voz no está en cuarentena. Campaña ante el contexto por el COVID 19. Asunción: CDE, 2020.

BANCO MUNDIAL. Remesas de trabajadores y compensación de empleados recibidas (\% del PIB). In: DATABANK. Washington: World Bank, 2018. Disponible en https://datos.bancomundial.org/indicador/BX.TRF.PWKR.DT.GD.ZS Acceso en agosto de 2020.

BATTHYANY, K. Las políticas y el cuidado en América Latina. Una mirada a las experiencias regionales. Santiago de Chile: CEPALAECID, 2015.

BATTHYANY, K; GENTA, N; SCAVINO, S. Análisis de género de las estrategias de cuidado infantil en Uruguay. Cadernos de pesquisa. v.47, n.163, p.292-319 jan./mar. 2017.

BORGEAUD-GARCIANDÍA, N. Entre desarrollo y fragmentaciones. Estudios del panorama del cuidado en Argentina. In: GUIMARÃES, N.A; HIRATA, H. (Comp.) El cuidado en América Latina. Buenos Aires: MEDIFÉ, 2020. p.27-70. 
CARRASCO, C. ¿La sostenibilidad de la vida humana, un asunto de mujeres? Revista Mientras Tanto, no 82. Barcelona: Icaria Editora, p.43-70,2001.

CECCHINI, S. M; MARTÍNEZ, R. Protección social inclusiva en América Latina. Una mirada integral, un enfoque de derechos. Santiago de Chile: CEPAL, 2011.

COMISIÓN ECONÓMICA PARA AMÉRICA LATINA. Observatorio de la Igualdad de Género. Santiago de Chile: OIG-CEPAL,2020. https://oig.cepal.org/es/documentos/la-pandemia-covid-19-profundiza-la-crisis-cuidados-america-latina-caribe acceso en julio de 2021.

COMISIÓN ECONÓMICA PARA AMÉRICA LATINA. Ficha técnica. In: Bases de datos y publicaciones estadísticas. Santiago de Chile: CEPALSTAT, 2019. Disponible en:.https://cepalstat-prod.cepal.org/portal/cepalstat/technical-sheet.html?lang=es\&indicator_id=35 acceso en julio de 2021.

DA SILVA, F.P. O Fim da Onda Rosa e o Neogolpismo na América Latina. Revista Sul-americana de Ciência Política, vol.4, n.2, p.165$178,2019$.

DARCY DE OLIVEIRA, R. Reengenharia do tempo.Rio de Janeiro: Editora Rocco, 2003.

DURÁN, M. El trabajo del cuidado en el marco macroeconómico. In: DURÁN, M. (Dir.) El trabajo de cuidado en América Latina y España. Madrid: Fundación. Carolina-CeALCI,2011.p.11-32.

ESPINO, A. Análisis de género en las políticas comerciales. In:TODARO, R; RODRÍGUEZ, R (Ed.) El género en la Economía, Santiago de Chile: ISIS/CEM.p.111-130, 2001.

FERRO, S.L. La cuestión del cuidado en el escenario post pandemia. ¿Hacia Estados del Cuidado o hacia Gilead? Revista Interdisciplinar em Cultura e Sociedade, v. 6, n. 2, p. 50 -71. São Luís: UFMA, 2020 b.

FERRO, S.L. Care's global crises, transnational migrations and remittances. Impacts in and from Latin America.Cidades, comunidades e territórios, n. 40,pp. 88 -102, 2020a.Lisboa:ISCTE-IUL.

FERRO, S.L. Aportes de la Economía del Cuidado para un sistema público de cuidados en América Latina.In: FERRO, S.L, THOMÉ, V.T.(Org.) Mulheres entre Fronteiras. Olhares Interdisciplinares desde o sul. Foz do Iguaçu: EDUNILA. 2019b.p.110-146.

FERRO, S.L. El cuidado como posibilidad de realización del paradigma de la universalidad y solidaridad en los sistemas de protección social. Una perspectiva histórica. Revista Interdisciplinaria de Estudios Sociales, n.17, p. 41-80. Bahía Blanca: CEISO, 2019a.

GÁLVEZ,T. Discriminación de género en el mercado laboral de América Latina: La brecha de ingresos 2001.In:TODARO, R; GODOY, L; ABRAMO, L. Trabajo decente y equidad de género en América Latina. Santiago de Chile: OIT, 2006.p.95-127.

GUIMARÃES, N.A; HIRATA, H. Realidades nacionales, desafíos latinoamericanos. In: GUIMARÃES, N.A; HIRATA, H. (Comp.) El cuidado en América Latina. Buenos Aires: MEDIFÉ, 2020.p.11-26.

GUIMARÃES, N.A; HIRATA, H; POSTHUMA, A. El cuidado: sus formas y actores. Reflexiones a partir del cas o de Brasil. In: GUIMARÃES, N.A; HIRATA, H. (Comp.) El cuidado en América Latina. Buenos Aires: MEDIFÉ, 2020. p.75-118.

HALPERIN DONGUI, T. Historia Contemporánea de América Latina. Madrid: Alianza Editorial, 2010.

HARARI, Y.N. Sapiens. Uma breve história da humanidade. Porto Alegre: L\&PM Editores, 2018.

HOBSBAWM, E. A era do capital, 1848-1875. São Paulo: Editora Paze Terra Ltda,2010.

INSTITUTO BRASILEIRO DE GEOGRAFIA E ESTATÍSTICA. Estatísticas de gênero. Indicadores sociais das mulheres no Brasil. Brasília: IBGE, 2018

JELIN, E. Pan y afectos. La transformación de las familias. Buenos Aires: Fondo de Cultura Económica, 2010.

ONU HABITAT. Estado de las ciudades. Nairobi: UN, 2010. Disponible em https://nacoesunidas.org/agencias/onuhabitat/ acceso agosto 2020.

ORGANIZACIÓN MUNDIAL DE LA SALUD. Innovaciones para abordar la violencia contra las mujeres en el contexto de COVID-19. Ginebra: UN, 2020. Disponible en https://www.paho.org/es/eventos/evento-oms-innovaciones-para-abordar-violencia-contramujeres-contexto-covid-19 acceso abril 2021 acceso agosto 2020.

PANIZZA, F. La marea rosa. Análise de Conjuntura OPSA. No. 8, p.1-16, 2006.

PARREÑAS, R. Servants of Globalization: Migration and Domestic Work. USA:Stanford University Press, 2015.

PÉREZ OROZCO, A. Amenaza tormenta: la crisis de los cuidados y la reorganización del sistema económico. Revista de Economía Crítica. No. 5, p. 7-37, 2006.

PICCHIO, A.Condiciones de vida, perspectivas, análisis económico y políticas públicas. Revista Economía Crítica. N. 7, p. 27-54, 2009. 
PICCHIO, A. La visibilidad analítica y política del trabajo de reproducción social”. In: CARRASCO, C.(ed.) Mujeres y Economía. Nuevas perspectivas para viejos y nuevos problemas. Barcelona: Editorial Icaria- Antrazyt, 2003.p.201-244.

PICCHIO, A. Un enfoque macroeconómico «ampliado» de las condiciones de vida. In: CARRASCO BENGOA, C. Tiempos, trabajos y género. Barcelona: Publicacions de la Universitat de Barcelona, 2001.p.15-40.

RAMA, G. Las políticas sociales en América Latina. Seminario: La teoría del desarrollo en los albores del siglo XXI. Santiago de Chile: CEPAL, 2001.

REPÚBLICA ARGENTINA. Medidas en materia de género y diversidad en el marco de la emergencia sanitaria. Buenos Aires: Ministerio de las Mujeres, Géneros y Diversidad, 2020. Disponible en https://www.argentina.gob.ar/generos/medidas-en-materia-degenero-y-diversidad-en-el-marco-de-la-emergencia-sanitaria acceso abril 2021.

REPÚBLiCA DEL PARAgUAY. Documento Marco para el Diseño de la Política Nacional de Cuidados en el Paraguay. Ministerio de la Mujer.Asunción: EUROSOCIAL, ONU MUJERES, $2019 . \quad$ Disponible http://www.mujer.gov.py/application/files/4715/6113/3467/Documento_Marco.Politica_Nacional_de_Cuidados_PY.pdf acceso abril 2021.

REPÚBLICA ORIENTAL DEL URUGUAY. Estrategias de los hogares en tiempos de COVID -19. Montevideo:In: SNIC, Montevideo, 2020b. Disponible en https://www.gub.uy/sistema-cuidados/comunicacion/noticias/estrategias-hogares-tiempos-covid-19-encuesta-sobreuso-del-tiempo-trabajo acceso abril 2021.

REPÚBLICA ORIENTAL DEL URUGUAY. Licencias por maternidad, paternidad, adopción y cuidados en la actividad pública y privada.Montevideo: In:SNIC, Montevideo, 2020a. Disponible en https://www.gub.uy/sistema-cuidados/ acceso abril 2021.

RODRÍGUEZ ENRIQUEZ, C. Programas de transferencias condicionadas de ingreso e igualdad de género ¿Por dónde anda América Latina? Santiago de Chile: CEPAL, 2011.

SAFFIOTI, H. I.B. Gênero, Patriarcado e Violência. São Paulo: Editora Fundação Perseu Abramo, 2004.

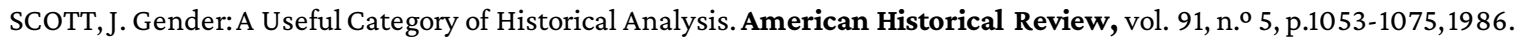

UN DESA. World Population Prospects. The 2019 revision. New York: UN, 2020.

UNFPA. Violencia basada en el género y COVID19. Montevideo: Fondo de las Naciones Unidas para la Población, sede Uruguay,2020. Disponible en Acceso abril 2021 en https://uruguay.unfpa.org/es/COVID19-y-violencia-basada-en-genero acceso abril 2021.

UN WOMEN. La pandemia en la sombra. New York, 2020. Disponible en https://www.unwomen.org/es/news/stories/2020/4/statement-ed-phumzile-violence-against-women-during-pandemic acceso abril 2021.

\begin{tabular}{ll}
\hline Funciones de colaboración ejercidas & \\
\hline Silvia Lilian Ferro: & $\begin{array}{l}\text { Concepción; Metodología; Validación; Curación de datos; Gestión del proyecto; Visualización; Análisis formal; Recursos; } \\
\text { Adquisición de financiación; Software; Investigación; Supervisión; Redacción(borrador original); Redacción (revisión y edición); }\end{array}$ \\
\hline
\end{tabular}

Información proporcionada por los(as) autores(as) según la Taxonomía de Funciones de Colaboración (CRediT) 